\title{
Consecuencias de los trabajos de Galileo en la ciencia posterior
}

\section{JUAN MANUEL RIVERA JUÁREZ ${ }^{1}$}

Revista EIA

ISSN 1794-1237

e-ISSN 2463-0950

Año XVIII/ Volumen 18/ Edición N.35

Enero-Junio de 2021

Reia35002 pp. 1-7

Publicación científica semestral Universidad EIA, Envigado, Colombia

\section{Para citar este artículo / To REFERENCE THIS ARTICLE /}

Rivera Juárez, J.M. (2021).

Consecuencias de los trabajos de

Galileo en la ciencia posterior.

Revista EIA, 18(35), Reia35002.

pp. 1-7.

https://doi.org/10.24050/reia.

v18i35.1455

\section{Autor de correspondencia:}

Rivera Juárez, J. M. (Juan Manuel): Universidad Autónoma de Zacatecas, México.

Correo electrónico:

jmrivera@uaz.edu.mx

Recibido: $07-08-2020$

Aceptado: 07-08-2020

Disponible online: 01-01-2021
1. Universidad Autónoma de Zacatecas. México

\section{Resumen}

Galileo es una figura clave en la historia de la ciencia, por ello muchos de sus conceptos y contenidos teóricos tienen un especial significado y trascendencia.

En Galileo, tal vez mejor que en ningún otro de los protagonistas de la revolución científica, puede apreciarse la dicotomía teórica del nacimiento de los nuevos y cruciales conceptos de la Física Clásica: muchas de sus opiniones y de sus conceptos son aún medievales, en tanto que otros son definitivamente modernos. Justamente esa dicotomía hace difícil apreciar el aporte real de Galileo en la formulación de los conceptos.

La mayoría de los trabajos realizados por Galileo tuvieron consecuencias para la ciencia posterior, en éste trabajo se analizarán solamente dos de estas consecuencias: las modificaciones del concepto de ímpetu, y la preparación de la primera ley del movimiento .

Palabras Claves: Historia de la Ciencia, Galileo, Física.

\section{Consequences of Galileo's work in later science}

\section{Abstract}

Galileo is an important personage in the history of science, that is why many of his concepts and technical contents have a special meaning and significance.

In Galileo's ideas, perhaps better than in any other of the protagonists of the scientific revolution, the theoretical dichotomy of the birth of the new and crucial concepts of Classical Physics can be appreciated: many of his opinions and concepts are still medieval while others are definitely modern. Precisely this dichotomy makes difficult to modify the real contribution of Galileo in the formulation of the concepts.

Most of the work carried out by Galileo had consequences for later science development, in this work only two of these consequences will be analyzed: the modifications of the concept of momentum, and the preparation of Newton's first law of motion.

Key Words: History of science, Galileo, Physics. 


\section{Introducción}

Fue justamente en la conceptualización del movimiento de los proyectiles, donde comenzó a desarrollarse la crítica a la doctrina aristotélica del movimiento, y de donde partieron finalmente, los conceptos que culminaron con la formulación del concepto de Inercia y de la Primera Ley de Newton.

La explicación aristotélica del movimiento de los proyectiles fue criticada inicialmente en el siglo II a.C. por Hiparco de Rodas, el astrónomo en desacuerdo con el planteamiento aristotélico sobre el movimiento de proyectiles plantea que el movimiento se da por medio de una fuerza, transmitida, al proyectil por el proyector. Esta fuerza absorbida por el proyectil se extingue gradualmente a medida que el proyectil se mueve, le llamó fuerza impresa, Franklin (1976).

Juan Filopón, reunió esta crítica y desarrolló la propuesta de Hiparco. Propuso que el instrumento de proyección imparte una potencia motriz al proyectil, y es esa fuerza impresa la que continúa moviéndolo, Gilson (1952).

Tuvo la intuición de visualizar la conservación de un movimiento sin la necesidad de un motor externo que actúe continuamente sobre el cuerpo, lo que constituyó un paso gigantesco en el análisis del movimiento.

La solución propuesta por Juan Filopón implica no la conservación del movimiento, sino la persistencia temporal de una fuerza motriz que mantiene el movimiento; lo que, si bien constituye un avance respecto a Aristóteles, está basado aún en un concepto fundamental de la física aristotélica: el movimiento debe ser producido por una fuerza, y la velocidad del cuerpo en movimiento es proporcional a dicha fuerza. Esta limitante fue una constante en la conceptualización del movimiento, salvo contadas excepciones, hasta bien entrado el siglo XVII.

Las ideas revolucionarias de Hiparco y Filopón se pueden considerar como los primeros esbozos de una teoría alternativa a los planteamientos aristotélicos sobre el movimiento de proyectiles, que luego fue desarrollada por Buridán.

Jean Buridán, al analizar el movimiento de los proyectiles propuso la teoría dinámica más influyente en los tres siglos siguientes, presenta la versión más acabada de la teoría del ímpetus -o fuerza impresa en un móvil-. De acuerdo con esta teoría, la conservación del movimiento de los proyectiles puede ser explicada por una especie de impulso, ímpetus, que el motor imprime en el cuerpo movido. La exposición del problema y la solución propuesta se encuentran en su obra Questiones super octo libros physicorum:

En cierta manera la fuerza motriz impresa conocida como ímpetus continuaba inscrita en la física aristotélica como una fuerza violenta que se oponía a la tendencia natural de los cuerpos hacia el reposo, pero esa fuerza no era ya producida por un motor externo, sino que se encontraba en el propio cuerpo en movimiento. Buridán dio así un paso decisivo en la historia del pensamiento; el respetado principio aristotélico todo lo que se mueve debe ser movido por algo, Aristóteles (1952). Había perdido su posición indiscutible hasta entonces.

La propuesta de Buridán fue un valioso intento de formulación cuantitativa que supera la noción de causa aristotélica: el ímpetus es concebido como una fuerza o poder poseído por el cuerpo sólo en razón de estar en movimiento, sin relación alguna con su naturaleza, su forma, o algún otro aspecto del cuerpo. 
Las características básicas de la teoría del ímpetus tal como fueron planteadas por Buridán se mantuvieron prácticamente sin modificación hasta el siglo XVII.

El principio de inercia constituye uno de los pilares fundamentales de la física clásica, en él están postulados todos los cambios esenciales en la concepción del mundo que marcó el fin de la física medieval y renacentista del siglo XVII. Desde entonces el concepto inercia se ha asimilado de tal manera que ha llegado a ser parte del lenguaje cotidiano, además su utilización se ha extendido a otros campos del conocimiento para designar en general la resistencia al cambio. Sin embargo, su significado es mucho más complejo, incluso tras la aparente sencillez de su enunciado se encuentra la prolongada y fecunda historia de su formación.

Las grandes etapas de su evolución se unen a los diversos modos de entender la naturaleza del movimiento -ya sea natural, cualidad o fuerza impresa o estado inercial-. De esta manera la historia del pensamiento físico desde Aristóteles a Newton se presenta en tres grandes etapas: la aristotélica, la del ímpetus y la matematizada.

El principio de inercia no puede inferirse directamente de la experiencia, -no es posible observarlo directamente en la naturaleza- sino a través de una suposición -abstracción- que sea acorde con lo observado. Al respecto, Alexandre Koyré, uno de los más representativos historiadores de la ciencia, plantea lo siguiente.

"El principio de la inercia no surgió ya elaborado del pensamiento de Descartes o de Galileo, como Atenea de la cabeza de Zeus", fue producto de un largo esfuerzo del pensamiento, Koyré (1980).

El principio de inercia es parte esencial del movimiento, el cual fue objeto de estudio desde la antigüedad. El primer estudio sistemático del movimiento corresponde a Aristóteles. Y fue la crítica a las teorías aristotélicas del movimiento, lo que constituyó el germen de la nueva ciencia del movimiento.

\section{Modificaciones del concepto de ímpetus}

La obra de Galileo se clasifica en tres periodos (el aristotélico, el de aceptación de la teoría del ímpetu y el de la maduración de la física moderna en su época). En el primero se aprecia la influencia de la tradición platónica y aristotélica; en el segundo se esfuerza por desarrollar de manera coherente y completa una dinámica de la fuerza impresa (o del ímpetus) al modo como la había desarrollado Benedetti en sus diferentes tratados. En los trabajos que comprenden esta segunda etapa se encuentran una vez más los tradicionales argumentos de sus predecesores parisienses, aunque condensados, sistematizados y un poco más claros. Desde esta perspectiva Galileo se muestra decididamente como un pensador anti-aristotélico.

Al comprobarse las consecuencias teóricas de la dinámica del ímpetus, la teoría llega a un callejón sin salida; será necesario que Galileo la abandone para lograr nuevas metas. Lo anterior Geymonat lo enuncia en el libro Galileo Galilei de la siguiente manera:

"Sobre todo la exigencia de matematizar la física le habría que el ambiguo concepto de «ímpetus» era insostenible y le convencería de la necesidad de constituir una nueva mecánica liberada tanto de los esquemas aristotélicos como de los de la física parisina" Geymonat.

En el tercer y último periodo de su pensamiento, Galileo supera la física de Aristóteles y también la Física del ímpetus y se eleva al nivel de la física matemática, 
en la que se va a manifestar su genialidad creadora, y también el nuevo rumbo de la ciencia.

La conceptualización galileana del movimiento fue así un resultado de la combinación de la nueva concepción del mundo expresamente contrapuesta a la filosofía y la física aristotélicas, con un método en el que la abstracción y el manejo de las hipótesis jugaron un papel decisivo a la par de la mate matización de la realidad y la experimentación. Desde Galilei, el movimiento es un concepto matemático tan plenamente válido como el triángulo o la pirámide.

Al abandonar la noción de ímpetus como causa interna del movimiento, permanecerá el término, Newton incluso lo utilizará también. Pero su significado será completamente transformado. De causa de movimiento, el ímpetus pasa a ser su efecto. El término ímpetus que era entendido como causa del movimiento que anima el móvil, se transforma en momento, es decir, producto de la velocidad por la masa, lo que es igual a la cantidad de movimiento. Se emplean los mismos términos tradicionales, pero su significado se va transformando.

De acuerdo con los principios generales de la filosofía natural de Galilei el universo es concebido como un conjunto material ordenado y sujeto a leyes físicas inmutables que pueden ser conocidas y expresadas matemáticamente. Con base en estos principios, estuvo en condiciones de formular una nueva concepción del movimiento, radicalmente distinta de la concepción aristotélica, y con ella varios de los principios básicos de la física clásica: la conservación y relatividad del movimiento, su composición, que hoy denominaríamos su carácter vectorial, así como el concepto de sistema inercial.

La idea del mundo en la obra de Galilei, aunque no exenta de términos y conceptos de la filosofía medieval, que con frecuencia recuerdan la concepción aristotélica de cosmos, no se apoyó en el concepto de «jerarquías» sino en la convicción de la existencia de relaciones regulares y necesarias de los cuerpos que integran el mundo material. En ella, la multiforme variedad de los elementos y naturalezas aristotélicas, con sus lugares naturales y movimientos propios, fue reemplazada por una concepción de corte platónico sobre la armonía geométrica del universo y el poder explicativo de las matemáticas. La explicación científica no se dio entonces en términos de acto y potencia, materia y forma, sino como una teoría matemática, verificable en la naturaleza, que rechazaba el concepto aristotélico de cambio sustancial, Shea (1983).

Galileo, a diferencia de sus predecesores, distingue cuidadosamente la condición de velocidad uniforme, que corresponde a una condición de equilibrio dinámico equivalente a la ausencia de fuerzas o causas que aceleren o retarden el movimiento. Al distinguir entre movimiento uniforme y movimiento acelerado, e identificar el peso como una fuerza que produce aceleración y que puede asimilarse a la fuerza con que se lanza un proyectil, Galilei, (1990). Sentó las bases de la dinámica y con ella la posibilidad del enunciado del principio de inercia.

Galileo cambia por completo el significado del concepto ímpetus.

"Galileo crea el concepto de ímpeto, donde se debe ver el germen de la fuerza generatriz de aceleración, concepto que llegará a ser "conatus" en el sentido de Huygens.” Dugas (1980). 


\section{Preparación de la primera ley del movimiento de New- ton}

La ley de inercia es un principio de extraordinaria importancia, porque implica una interpretación general de la naturaleza, es decir, supone una concepción nueva de la misma realidad física. El movimiento ahora es un estado y no un proceso como para la física anterior. Movimiento y reposo son colocados al mismo nivel ontológico. El paso de un estado a otro ha de requerir no ya la "evidencia" de la física aristotélica, sino una causa o "fuerza" que necesariamente ha de concebirse como exterior y ajena al móvil que, en sí, es inerte.

Ahora bien, para la ciencia moderna la acción de una fuerza no produce un movimiento, sino una aceleración. Por ello, Galileo reduce ya la acción de una fuerza a un gradual aumento de la velocidad, al acumularse los incrementos de la velocidad misma. La fuerza es entonces una secuencia continua de impulsos instantáneos que se añaden unos a otros. Pero esto solo es posible con la asunción, al menos implícita, del principio de inercia, pues presuponer el principio de inercia es concebir la fuerza ya no como una cualidad o una propiedad interna al móvil, sino como un empuje, una actividad externa a la que se considera como la responsable de la aceleración del movimiento.

Como es del conocimiento de todos, Galileo no llegará a enunciar expresamente el principio de inercia. Nunca afirmará la real consecuencia eterna del movimiento rectilíneo.

"Es imposible que ningún móvil tenga por naturaleza el principio de moverse en línea recta; es decir, hacia donde es imposible llegar, al no existir un término predefinido; pues la naturaleza, como dice el mismo Aristóteles, no comienza a hacer aquello que no puede ser hecho, ni comienza a moverse hacia donde es imposible llegar". Galilei (1968).

Es paradójico que Galilei no haya formulado expresamente el principio de inercia; sin embargo, como bien lo afirma Koyré (1973), toda su mecánica se encuentra implícitamente basada en él.

La ejemplificación más clara de esta convergencia entre la física galileana y la física inercial es el principio de conservación del movimiento, que muchas veces ha sido confundido con el principio de inercia. El planteamiento final de la conservación del movimiento como lo propuso Galilei tiene un extraordinario parecido con el principio de inercia, pero no cumple aún dos condiciones básicas para una formulación completa: que se conserve tanto el reposo como el movimiento, y que en éste la velocidad se conserve tanto en magnitud como en dirección, cualquiera que sea el estado y la dirección inicial del movimiento.

Si bien es cierto que Galilei no formuló el principio de inercia es indudable que los elementos del movimiento inercial que se encuentran comprendidos en su obra conducen a descubrir una inercia restringida, condicionada siempre por la acción del peso de los cuerpos.

Es cierto que Galilei no logró superar el último obstáculo que halló en el análisis del movimiento -el carácter pesado de los cuerpos- y tampoco formuló el principio de inercia, pero su excepcional comprensión de la dinámica le permitió reconocer y proponer la diferencia radical entre el movimiento uniforme y el movimiento acelerado; asimismo identificó la componente inercial en los movimientos reales. Por eso no es aventurado afirmar que en el análisis galileano del movimiento y en el tratamiento matemático que ofrece en los Discorsi se sitúa la fundamentación dinámica del princi- 
pio de inercia, a tal punto que el mismo Newton creyó ver allí sus dos primeras leyes del movimiento.

Con respecto a está indecisión a la hora de definir o establecer algunos criterios o planteamientos por parte de Galileo, ya sea por qué no supo, no pudo o no quiso hacerlo, Koyre señala lo siguiente:

"Si en verdad fracasó Galileo en su tarea-de llagar al principio de inercia-fue por qué, al contrario de Descartes, no supo o no pudo librarse del hecho ni aceptar la consecuencia inevitable de la mate matización de lo real: la geometrización completa del espacio, es decir, la infinitud del universo y la destrucción del cosmos" Koyré (1980).

A pesar de que la finitud de su concepción del universo supuso una barrera infranqueable, que hizo que Galileo no llegara a la profundidad del principio de inercia, hay razones suficientes para considerar, como lo plantea Jammer, que preparó el camino de la formulación newtoniana.

"Galileo fue quien preparó el terreno para esta conclusión que forma la base de las dos primeras leyes del movimiento formuladas por Newton. Fue el concepto del movimiento libre de fuerzas el que contribuye al presupuesto de la concepción newtoniana de fuerza" Jammer (1971).

\section{Comentarios finales}

La importancia histórica del concepto inercia en el desarrollo de la mecánica es innegable. Las revoluciones científicas no fueron producidas por un cambio en el concepto de inercia, sino por rupturas de otro tipo. Fue necesario el concurso de las mentes más lúcidas del mundo occidental durante dos mil años para superar la dinámica aristotélica y de ese modo generar las condiciones para que se pudiera enunciar el principio de inercia y sentar las bases de la mecánica clásica.

Las propuestas teóricas de Galileo no contaron con una aceptación generalizada en su tiempo, pese a que estaban ya dadas las condiciones para que la nueva ciencia del movimiento y sus fundamentos influyeran en el ambiente científico de la época y era lógico que así sucediera: la idea de un movimiento sin un motor externo se había abierto camino con la física del ímpetus, pero la conservación del movimiento en ausencia de toda fuerza motriz parecía absurda y contra natura.

\section{Referencias}

[Aristóteles, Physica, s. IV a. C. Hardie, R.P. \& Gaye, R. K. (1952). Physics, The Works of Aristotle, Great Books of the Western World. Chicago: Enciclopedia Británica, pp. 326.

Dugas, R. (1954). La mecánica au XVII esiécle, París: Vrin, pp. 605.

Franklin, A. (1976). Principle of inertia in the middle ages. Am. J. Phys. 44, pp. 529-545. https://doi.org/10.1119/1.10392

Galilei, G. (1990). Discorsi e dimostrazioni matematiche attinenti alla mechanica ed i movimenti locali. En Elsevirii, L., Edizione a cura di E. Giusti. Torino: Giulio Einaudi Editore. 
Galilei, G. (1968) Opere, Tomo VII, “Dialogo sopra due massimi sistema del mondo”, 1aㅡ Jornada, pp. 43.

Geymonat, L. (1986). Galileo Galilei. Barcelona: Península

Gilson, E. (1952). La Philosophie au Moyen Age, París, Payot. Pacios A. y Caballero, S. (1985) La filosofía de la Edad Media, Madrid: Gredos.

Jammer, M. (1979). Stonia del concetto di forza, Milano: ed. Feltrinelli, 2ª edizione, pp. 114.

Koyré, A. (1980). Estudios Galileanos Madrid: Siglo XXI, pp. 152-199.

Shea W. R. (1983). La revolución intel 